\title{
Mortalidade cardiovascular e impacto de técnicas corretivas de subnotificações e óbitos mal definidos
}

\author{
David Arnaud Soares ${ }^{1}$ e Maria Jacirema Gonçalves ${ }^{2}$
}

Como citar Soares DA, Gonçalves MJ. Mortalidade cardiovascular e impacto de técnicas corretivas de subnotificações e óbitos mal definidos. Rev Panam Salud Publica. 2012;32(3):199-206.

RESUMO Objetivo. Identificar o impacto de técnicas corretivas na tendência das taxas de mortalidade por doenças cardiovasculares, doenças isquêmicas do coração e doenças cerebrovasculares, em Manaus (AM), Brasil, no período entre 1980 e 2007.

Métodos. Os dados foram obtidos no Departamento de Informática do Sistema Único de Saúde, considerando-se os seguintes passos: (1) redistribuição proporcional dos registros de óbitos com idade e/ou sexo ignorados; (2) redistribuição dos óbitos mal definidos dentre os de causa conhecida; (3) tratamento e realocação dos chamados "códigos-lixo" em cardiologia; $e$, (4) correção dos sub-registros de óbitos por técnicas indiretas. Registros tratados nos passos 1, 2 e passos 3 e 4 agregados geraram as taxas base, taxas ajustadas $e$ taxas ajustadas e corrigidas, respectivamente, as quais foram analisadas por sexo e faixa etária; posteriormente, foram padronizadas pelo método direto. A regressão linear simples foi utilizada para análise de tendência.

Resultados. Os dados brutos do Sistema de Informação sobre Mortalidade subestimavam as taxas de mortalidade por doenças do coração, principalmente a partir do ano 2000. Quanto à tendência temporal, as taxas ajustadas e corrigidas, comparadas às taxas base, apontam: atenuação da tendência de queda para as doenças cardiovasculares como um todo; acentuação da tendência de queda para as doenças isquêmicas do coração; e estabilidade nas taxas para as doenças cerebrovasculares no período.

Conclusões. Com essa correção, foi possível identificar um excesso de óbitos que não havia sido computado, gerando taxas de mortalidade ajustadas e corrigidas mais confiáveis.

Palavras-chave Doenças cardiovasculares; estatísticas vitais; mortalidade; sub-registro; registros de mortalidade; sistemas de informação; Brasil.

As Doenças e Agravos Não Transmissíveis (DANT) são as principais causas de óbito no Brasil e no mundo, principalmente como reflexo do aumento da

\footnotetext{
Hospital Universitário Getúlio Vargas, Universidade Federal do Amazonas, Manaus, Amazonas, Brasil.

2 Escola de Enfermagem de Manaus, Universidade Federal do Amazonas, Manaus, Amazonas, Brasil. Enviar correspondência para Maria Jacirema Gonçalves, jaciremagoncalves@gmail.com
}

população idosa (1). As doenças cardiovasculares (DCV) compõem parcela desse grupo e representaram um terço dos óbitos globais no ano de 2004 (2). Isso se deu não só por fatores demográficos, mas também pela mudança no perfil epidemiológico populacional, a chamada "transição epidemiológica" (3). Esse fenômeno, mais que uma mudança obrigatória e linear, é heterogêneo, refletindo o grau de desenvolvimento urbano e o momento demográfico da população local (4).

No entanto, tal qual em países desenvolvidos, estudos de tendência publicados no Brasil apontam queda das taxas de mortalidade por DCV, principalmente nas capitais, cuja prioridade nos estudos se deve a maior confiabilidade nas notificações feitas nas bases de dados nacionais, por concentrarem menor percentual de óbitos com causas 
mal definidas e por estarem menos sujeitas ao sub-registro de óbitos $(5,6)$.

As doenças isquêmicas do coração (DIC), somadas às doenças cerebrovasculares $(\mathrm{DCbV})$, representam mais de $60 \%$ dos óbitos por DCV no Brasil (7).

Embora a confiabilidade dos dados secundários de óbitos seja questionada, principalmente para as regiões Norte e Nordeste do Brasil (8), alguns estudos de tendência de mortalidade por doenças do aparelho circulatório utilizam dados brutos do Sistema de Informação sobre Mortalidade (SIM), sem uso de métodos de correção. Eles efetuam, basicamente, a distribuição proporcional dos óbitos por causas mal definidas (9) entre os capítulos com óbitos definidos (10-12).

Poucas análises no Brasil têm utilizado técnicas de correção de sub-registro de óbitos (13-16). Estudos que tenham o município como unidade de análise não foram detectados, porém, são desejáveis, já que podem permitir que as técnicas corretivas aplicadas aos dados secundários de óbitos sejam utilizadas como ferramenta de auxílio à gestão local.

Manaus, capital do Estado do Amazonas, maior município da região Norte do Brasil em termos econômicos e populacionais, segundo o Instituto Brasileiro de Geografia e Estatística (IBGE) (17), carece de estudos quanto à tendência da mortalidade por DCV e seus subgrupos. Observa-se que, somente a partir do ano de 2003, as DCV assumiram o topo entre as causas de morte na cidade, enquanto, em nível nacional, isso já era realidade desde o início da série histórica do SIM, em 1979. Entretanto, uma análise mais acurada dessa tendência requer a devida correção dos dados secundários.

O presente trabalho teve por objetivo identificar o impacto do uso de técnicas corretivas nas curvas de tendência das taxas de mortalidade por DCV, DIC e $\mathrm{DCbV}$, em Manaus, no período de 1980 a 2007, segundo notificações do SIM.

\section{MATERIAIS E MÉTODOS}

Foi realizado um estudo de tendência temporal de mortalidade por DCV na cidade de Manaus, a partir das taxas de mortalidade por DCV, DIC e DCbV, no período de 1980 até 2007, estratificadas por sexo e faixa etária.

O Departamento de Informática do Sistema Único de Saúde (DATASUS)
(18) é a fonte de dados populacionais e de óbitos - os últimos são considerados, mais especificamente, pelo SIM.

Nas etapas de ajuste e correção dos óbitos, utilizaram-se os métodos de correção adotados no Projeto Estimativa de Carga de Doenças no Brasil (19). Para correção da sub-registro de óbitos, utilizaram-se as técnicas de Brass (20) e Preston et al. (21), descritas em publicação das Nações Unidas (22).

Dos dados de mortalidade em Manaus, filtraram-se os óbitos de 1980 até 1995, com base na Classificação Internacional de Doenças ( CID 9), selecionando os óbitos por DCV (códigos 390 a 459), DIC (códigos 410 a 414) e DCbV (códigos 430 a 438). A estes, somaram-se os registros filtrados, de 1996 a 2007, com base na CID 10, com os códigos I10 a I82.9, I20 a I25 e I60 a I69.

Estratificaram-se os dados por sexo e faixa etária: menor que 5 anos; 7 faixas com intervalo de 10 anos, de 5 até 75 anos; e mais de 75 anos.

Inicialmente, os registros de óbitos foram tratados com sexo e/ou idade ignorada, por meio da redistribuição proporcional utilizada pela Organização Pan-Americana de Saúde (23). Em seguida, foram calculadas as taxas de mortalidade brutas, por sexo e faixa etária (número de óbitos/população $\times$ 100.000), as quais foram padronizadas pelo método direto (24), tendo como padrão a população brasileira do censo demográfico de 1991, por ser o dado censitário mais próximo da mediana do período do estudo (25), o que gerou as "taxas base".

Na sequência, realizou-se o tratamento dos óbitos mal definidos (códigos 780799, na CID 9, e códigos R00-R99, na CID 10). Assumiu-se que o grau de cobertura dos óbitos, em razão de causas externas, era próximo de $100 \%$. Desse modo, tais notificações foram excluídas, levando em conta apenas as causas de morte denominadas "naturais". Aqui também se utilizou a redistribuição proporcional dos óbitos (23), para os três grupos de causas de óbitos. Como exemplo desse procedimento, apresentou-se o cálculo para as DCV: óbitos por DCV ajustados = óbitos por DCV base + ((óbitos por DCV base/óbitos definidos) $\times$ óbitos mal definidos). Os primeiros parênteses contêm a fórmula que calcula a distribuição proporcional dos óbitos por DCV entre os óbitos mal definidos. Os segundos parênteses multiplicam essa proporção pelo número de óbitos mal definidos, gerando o número de óbitos incorporado ao arquivo DCV base, para se obter a correção proposta. Os óbitos definidos foram obtidos pela fórmula: óbitos definidos = (total de óbitos - óbitos mal definidos - óbitos por causas externas). A partir dos ajustes, calcularam-se as novas taxas de mortalidade brutas e padronizadas pelo método direto, gerando o que se denominou "taxas ajustadas".

Na sequência, procedeu-se à distribuição dos "códigos-lixo" em cardiologia (26). Um percentual desses óbitos deve ser realocado como óbitos por DIC, em faixas etárias específicas. Como os óbitos por DCbV seriam superestimados e não subestimados, não houve necessidade de correção para esse grupo de causas de óbitos (26).

Para o tratamento dos códigos-lixo em cardiologia, adotaram-se o estudo australiano de carga de doenças (27) e o estudo de carga de doenças no Brasil (19). Como a presente série histórica contém dados codificados pela CID 9 e pela CID 10, foi necessário encontrar correspondência entre os códigos-lixo de ambas as CID, valendo-se da lista de conversão publicada pelo Ministério da Saúde da Nova Zelândia (28).

$\mathrm{Na}$ última etapa, aplicaram-se aos dados de óbitos da etapa anterior os fatores $\mathrm{K}$ calculados anualmente para cada sexo, segundo as técnicas indiretas adotadas por Brass (20) e Preston et al. (21). $\mathrm{O}$ fator $\mathrm{K}$ se refere ao multiplicador para corrigir os registros de óbitos. Quando a completude (C) foi inferior a 1,0, indicava que houve sub-registro no ano estudado. Tais métodos retornam a taxa de crescimento populacional (r). Esse dado, comparado à taxa de crescimento real no ano, foi utilizado para selecionar o método a empregar, quando ambos indicavam incompletude de dados $(C<1,0)$. Nos anos em que nenhuma das técnicas apontava $C<1,0$, não se aplicou a correção. Quando somente uma técnica apontava $C<1,0$, aplicou-se o fator K. Quando as duas técnicas apontavam $C<1,0$, escolheu-se a que apresentava taxas de crescimento populacional mais próximas das taxas reais.

A partir da correção dos óbitos pelos fatores $K$, calcularam-se as novas taxas de mortalidade brutas e padronizadas para as três causas de óbitos por sexo, gerando as "taxas ajustadas e corrigidas". 
Dados contendo as taxas de mortalidade padronizadas para a população residente em Manaus para ambos os sexos, nos três níveis do estudo (taxas base, taxas ajustadas e taxas ajustadas e corrigidas), foram analisados por regressão linear simples, a partir do teste de assimetria e curtose (Shapiro-Wilk), para detectar a normalidade. As tendências lineares são representadas na função $y=b x+a$, na qual $b$ reflete a inclinação da reta, $x$ é o ano de análise com o valor mínimo de 0 para 1980 e máximo de 27 para o ano 2007, e $a$ é a taxa calculada para o primeiro ano da série. A variância anual (\%v) é obtida de b/a $x$ 100. Avaliou-se o ajuste da regressão pelo coeficiente $\mathrm{R}^{2} \mathrm{e}$ pela análise dos resíduos. A significância estatística foi considerada ao nível de $5 \%$. Escolheu-se o método de regressão linear simples, pois os dados se adequaram a esse tipo de análise e porque se desejava medir a tendência, e não analisar flutuações locais ou sazonalidade.

Para analisar os dados, corrigir propostas e adequar os bancos de mortalidade e das populações, empregaram-se os seguintes programas: Microsoft Access $^{\circledR}$, Microsoft Excel ${ }^{\circledR}$, programa estatístico R, versão 2.13.0 (r-project), e Stata, versão 9.0 (StataCorp).

O projeto foi aprovado no Comitê de Ética em Pesquisa (CEP) da Universidade Federal do Amazonas (UFAM), com CAAE n. ${ }^{\circ}$ 0329.0.115.000-09.

\section{RESULTADOS}

Tendo por base o estudo de Mathers et al. (27), o percentual de óbitos alocados como DIC, oriundos daqueles registrados como Insuficiência Cardíaca (código 428, na CID 9, e I50, na CID 10), foi de $70 \%$ para a faixa etária dos 30 até os 59 anos, e de $60 \%$ para 60 anos ou mais. Para os demais códigos-lixo, o percentual foi $75 \%$ na primeira faixa etária e de $80 \%$ para a segunda.

A Tabela 1 mostra os códigos-lixo em cardiologia, codificados pela CID 10 , e sua respectiva correspondência na CID 9.

Pela inexistência de códigos da CID 9 com cinco dígitos, desconsideraram-se os códigos 427.8.9, 427.3.1 e 427.4.1 na conversão para DIC. Das codificações com quatro dígitos (427.8, 427.3 e 427.4), localizaram-se apenas 53 registros do total de 14.693 óbitos por DCV entre 1980 e 1995. A incorporação desse pe-

TABELA 1. Lista de códigos-lixo em cardiologia, CID 9 e CID 10

\begin{tabular}{lll}
\hline \multicolumn{1}{c}{ Doença/sintoma } & CID-9 & CID-10 \\
\hline Parada cardíaca & 427.5 & 146 \\
Arritmia ventricular por reentrada & 427.8 .9 & 147.0 \\
Taquicardia supraventricular & 427.0 & 147.1 \\
Taquicardia ventricular & 427.1 & 147.2 \\
Taquicardia paroxística não específica & 427.2 & 147.9 \\
Flutter e fibrilação atrial & 427.3 .1 & 148 \\
Flutter e fibrilação ventricular & 427.4 .1 & 149.0 \\
Arritmia cardíaca não especificada & 427.9 & 149.9 \\
Insuficiência cardíaca & 428 & 150 \\
Miocardite não especificada & 429.0 & 151.4 \\
Degeneração do miocárdio & 429.1 & 151.5 \\
Doença cardiovascular não especificada & 429.2 & 151.6 \\
Doença não especificada do coração & 429.9 & 151.9 \\
Aterosclerose generalizada e não especificada & 440.9 & 170.9 \\
\hline
\end{tabular}

Fontes: Gadelha et al. (15); Mathers et al. (22). Adaptado pelos autores a partir das fontes originais. CID: Classificação Internacional de Doenças.

TABELA 2. Fatores $\mathrm{K}$ para correção de sub-registro de óbitos e demais dados retornados pelas técnicas de Brass (16) e Preston et al. (17)

\begin{tabular}{|c|c|c|c|c|c|c|c|c|}
\hline \multirow[b]{2}{*}{ Ano } & \multicolumn{4}{|c|}{ Feminino } & \multicolumn{4}{|c|}{ Masculino } \\
\hline & C & $\mathrm{K}$ & $r$ & Método & C & $\mathrm{K}$ & $r$ & Método \\
\hline 1980 & 0,9694 & 1,0315 & 0,0412 & Preston & 0,9898 & 1,0103 & 0,0368 & Preston \\
\hline 1981 & 0,9442 & 1,0591 & 0,0415 & Preston & 0,9049 & 1,1051 & 0,0380 & Brass \\
\hline 1982 & 0,8813 & 1,1347 & 0,0421 & Preston & 0,9773 & 1,0232 & 0,0387 & Brass \\
\hline 1983 & - & - & - & - & - & - & - & - \\
\hline 1984 & 0,9493 & 1,0534 & 0,0418 & Brass & - & - & - & - \\
\hline 1985 & 0,9941 & 1,0060 & 0,0423 & Brass & 0,9972 & 1,0028 & 0,0396 & Brass \\
\hline 1986 & - & - & - & - & - & - & - & - \\
\hline 1987 & - & - & - & - & - & - & - & - \\
\hline 1988 & - & - & - & - & - & - & - & - \\
\hline 1989 & - & - & - & - & - & - & - & - \\
\hline 1990 & - & - & - & - & - & - & - & - \\
\hline 1991 & - & - & - & - & - & - & - & - \\
\hline 1992 & 0,9146 & 1,0934 & 0,0414 & Brass & 0,9435 & 1,0599 & 0,0385 & Brass \\
\hline 1993 & 0,9974 & 1,0026 & 0,0422 & Brass & - & - & - & - \\
\hline 1994 & - & - & - & - & - & - & - & - \\
\hline 1995 & - & - & - & - & - & - & - & - \\
\hline 1996 & 0,8727 & 1,1459 & 0,0386 & Brass & 0,9487 & 1,0541 & 0,0359 & Brass \\
\hline 1997 & 0,9223 & 1,0842 & 0,0388 & Brass & 0,9863 & 1,0139 & 0,0361 & Brass \\
\hline 1998 & 0,9023 & 1,1083 & 0,0387 & Brass & - & - & - & - \\
\hline 1999 & 0,9235 & 1,0829 & 0,0388 & Brass & - & - & - & - \\
\hline 2000 & 0,7522 & 1,3294 & 0,0349 & Brass & 0,8600 & 1,1628 & 0,0332 & Brass \\
\hline 2001 & 0,7624 & 1,3117 & 0,0350 & Brass & 0,8542 & 1,1707 & 0,0338 & Brass \\
\hline 2002 & 0,7433 & 1,3454 & 0,0351 & Brass & 0,8197 & 1,2200 & 0,0330 & Brass \\
\hline 2003 & 0,7182 & 1,3924 & 0,0350 & Brass & 0,8385 & 1,1926 & 0,0335 & Brass \\
\hline 2004 & 0,7652 & 1,3068 & 0,0353 & Brass & 0,8730 & 1,1454 & 0,0334 & Brass \\
\hline 2005 & 0,7686 & 1,3010 & 0,0352 & Brass & 0,8388 & 1,1922 & 0,0334 & Brass \\
\hline 2006 & 0,7700 & 1,2987 & 0,0352 & Brass & 0,8283 & 1,2073 & 0,0333 & Brass \\
\hline 2007 & $0,7590^{a}$ & $1,3175^{b}$ & - & b & $0,8136^{a}$ & $1,2291^{b}$ & - & b \\
\hline
\end{tabular}

C: completude de óbitos; $\mathrm{K}$ : fator de correção (multiplicador); r: taxa de crescimento populacional.

a Calculado a partir de $\mathrm{K}(\mathrm{C}=1 / \mathrm{K})$.

b Média dos dois métodos.

queno número de registros foi descartada, por eles incluírem subitens não previstos como códigos-lixo (29).

Foram detectados e redistribuídos 1.272 óbitos, antes registrados como Insuficiência Cardíaca, para as DIC de 1980 a 1995 (CID 9) e 744 de 1996 a 2007 (CID 10). O percentual de acréscimo às DIC, em cada período, foi, respectivamente, $39 \%$ e $25 \%$. Para os demais códigos-lixo, foram incorporados 1.434 óbitos às DIC de 1980 a 1995 (CID 9) e 223 de 1996 a 2007, correspondendo, respectivamente, a $43 \%$ e $7 \%$ de acréscimo.

A Tabela 2 apresenta os fatores $\mathrm{K}$ para correção de sub-registro de óbitos, a C 
FIGURA 1. Taxas de mortalidade padronizada por doenças cardiovasculares, em Manaus, no período de 1980 a 2007. (A) Taxa de mortalidade padronizada masculina; (B) taxa de mortalidade padronizada feminina
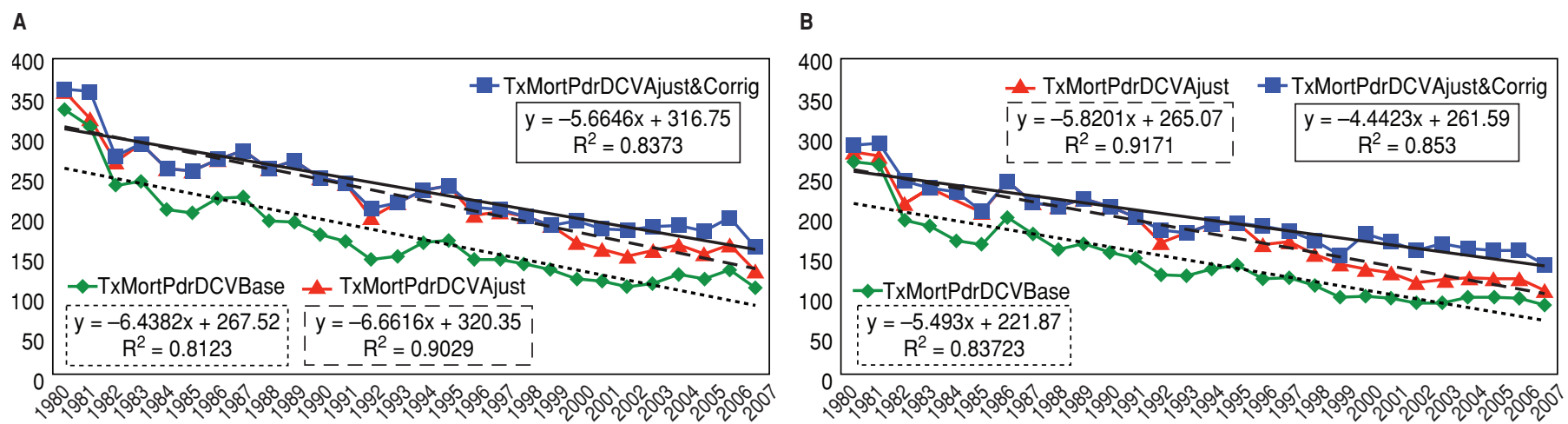

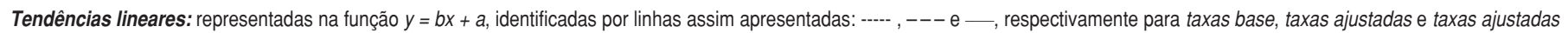
e corrigidas.

TxMortPdrDVC: taxa de mortalidade padronizada por doenças cardiovasculares; base: taxas calculadas sem correção dos registros de óbitos; Ajust: taxas ajustadas com redistribuição dos registros de óbitos mal definidos; Ajust\&Corrig: taxas calculadas com os ajustes prévios mais correção de sub-registro de óbitos.

FIGURA 2. Taxas de mortalidade padronizada por doenças isquêmicas do coração, em Manaus, no período de 1980 a 2007. (A) Taxa de mortalidade padronizada masculina; (B) taxa de mortalidade padronizada feminina

A

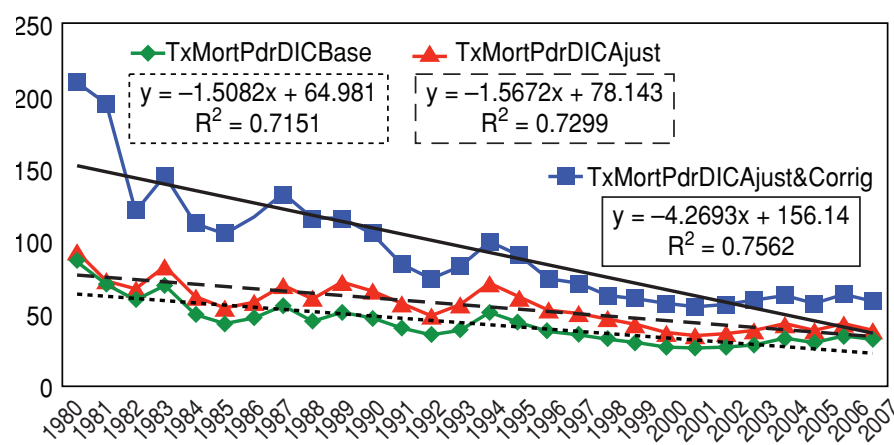

B

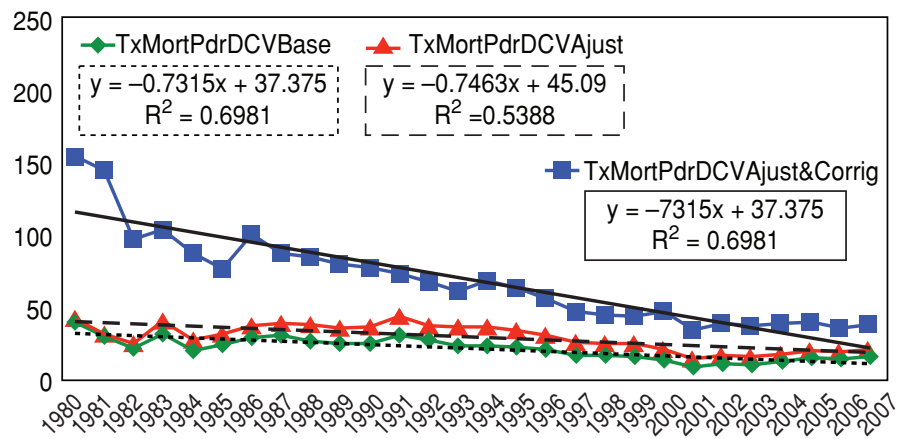

Tendências lineares: representadas na função $y=b x+a$, identificadas por linhas assim apresentadas: ---- , --- e - —, respectivamente para taxas base, taxas ajustadas e taxas ajustadas e corrigidas.

TxMortPdrDIC: taxa de mortalidade padronizada por doenças isquêmicas do coração; Base: taxas calculadas sem correção dos registros de óbitos; Ajust: taxas ajustadas com redistribuição dos registros de óbitos mal definidos; Ajust\&Corrig: taxas calculadas com os ajustes prévios mais correção de sub-registro de óbitos.

de óbitos e o valor de r retornado pelos métodos demográficos indiretos utilizados $(20,21)$. Como, na última década do estudo, as taxas de crescimento pela técnica de Preston et al. (21) mostraram-se elevadas, descartou-se a utilização do método nesse período. Os dados de 2007 foram tratados pela média das duas técnicas $(14,30)$, visto que a não correção geraria queda abrupta nas taxas ajustadas e corrigidas.

Os métodos usados para correção do sub-registro de óbitos no primeiro ano de vida exigiriam a informação do número de nascidos vivos, dado não disponível no Sistema de Informação sobre Nasci- dos Vivos (SINASC) até o ano de 1993. Fez-se a opção por não corrigir a primeira faixa populacional, sendo observado que, após a padronização das taxas de mortalidade por DCV, DIC e DCbV, anualmente, antes e após as correções propostas neste estudo, o impacto dessa decisão foi pequeno, devido à baixa prevalência na faixa etária de 0 a 5 anos.

As Figuras 1, 2 e 3 mostram respectivamente as taxas de mortalidade padronizadas para DCV, DIC e DCbV, entre homens e mulheres. Cada figura mostra as taxas padronizadas, apresentando-se os dados sem correção (taxas base); os ajustados quanto aos óbitos mal defi- nidos (taxas ajustadas); e a incorporação dos códigos-lixo redistribuídos e correção do sub-registro de óbitos (taxas ajustadas e corrigidas).

Para as DCV, as figuras mostram tendência de declínio das taxas de mortalidade. Há que se observar que, tanto entre homens, quanto entre mulheres, as taxas com correção de sub-registro de óbitos apontam menor inclinação da tendência (Tabela 3). Nota-se que as taxas ajustadas apenas com a distribuição dos óbitos mal definidos, têm, para ambos os sexos, incremento na tendência de declínio, com valores de $b$ maiores que nas taxas base. Os valores de $\mathrm{R}^{2}$ para as $\mathrm{DCV}$, 
FIGURA 3. Taxas de mortalidade padronizada por doenças cerebrovasculares, em Manaus, no período de 1980 a 2007. (A) Taxa de mortalidade padronizada masculina; (B) taxa de mortalidade padronizada feminina

A

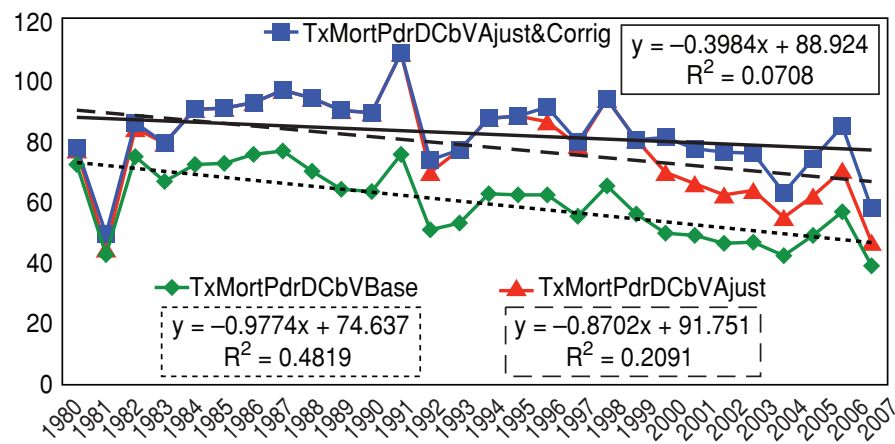

B

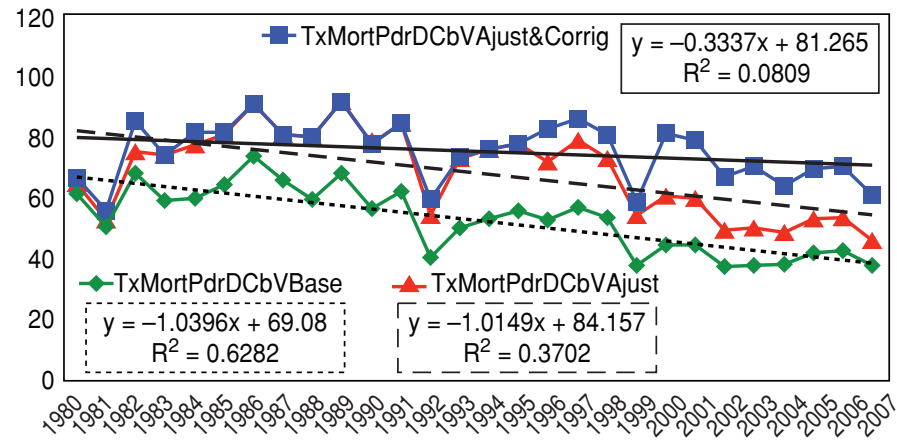

Tendências lineares: representadas na função $y=b x+a$, identificadas por linhas assim apresentadas: ----- ,--- e — , respectivamente para taxas base, taxas ajustadas e taxas ajustadas e corrigidas.

TxMortPdrDCbV: taxa de mortalidade padronizada por doenças cerebrovasculares; Base: taxas calculadas sem correção dos registros de óbitos; Ajust: taxas ajustadas com redistribuição dos registros de óbitos mal definidos; Ajust\&Corrig: taxas calculadas com os ajustes prévios mais correção de sub-registro de óbitos.

TABELA 3. Tendências das taxas de mortalidade padronizadas por DCV, DIC e DCbV em Manaus, 1980 a 2007

\begin{tabular}{|c|c|c|c|c|c|c|c|c|c|c|c|c|c|}
\hline & & \multicolumn{6}{|c|}{ Masculino } & \multicolumn{6}{|c|}{ Feminino } \\
\hline & & $a=y^{1980}$ & $y^{2007}$ & $b$ & $\% v$ & $\mathrm{R}^{2}$ & $P$ & $a=y^{1980}$ & $y^{2007}$ & $\mathrm{~b}$ & $\% v$ & $\mathrm{R}^{2}$ & $P$ \\
\hline & Base & 267,52 & 93,69 & $-6,4382$ & $-2,41$ & 0,8123 & $<0,01$ & 221,87 & 73,56 & $-5,4930$ & $-2,48$ & 0,8372 & $<0,01$ \\
\hline \multirow[t]{3}{*}{ DCV } & Ajust. & 320,35 & 140,49 & $-6,6616$ & $-2,08$ & 0,9029 & $<0,01$ & 265,07 & 107,93 & $-5,8201$ & $-2,20$ & 0,9171 & $<0,01$ \\
\hline & Ajust.\&Corrig. & 316,75 & 163,81 & $-5,6646$ & $-1,79$ & 0,8373 & $<0,01$ & 261,59 & 141,65 & $-4,4423$ & $-1,70$ & 0,8530 & $<0,01$ \\
\hline & Base & 64,98 & 24,26 & $-1,5082$ & $-2,32$ & 0,7151 & $<0,01$ & 37,38 & 17,62 & $-0,7315$ & $-1,96$ & 0,6981 & $<0,01$ \\
\hline \multirow[t]{3}{*}{ DIC } & Ajust. & 78,14 & 35,83 & $-1,5672$ & $-2,01$ & 0,7299 & $<0,01$ & 45,09 & 24,94 & $-0,7463$ & $-1,66$ & 0,5388 & $<0,01$ \\
\hline & Ajust.\&Corrig. & 156,14 & 40,87 & $-4,2693$ & $-2,73$ & 0,7562 & $<0,01$ & 118,98 & 30,38 & $-3,2815$ & $-2,76$ & 0,8335 & $<0,01$ \\
\hline & Base & 74,64 & 48,25 & $-0,9774$ & $-1,31$ & 0,4819 & $<0,01$ & 69,08 & 41,01 & $-1,0396$ & $-1,50$ & 0,6282 & $<0,01$ \\
\hline \multirow[t]{2}{*}{ DCbV } & Ajust. & 91,75 & 68,26 & $-0,8702$ & $-0,95$ & 0,2091 & 0,01 & 84,16 & 56,75 & $-1,0149$ & $-1,21$ & 0,3702 & $<0,01$ \\
\hline & Ajust.\&Corrig. & 88,92 & 78,17 & $-0,3984$ & $-0,45$ & 0,0708 & 0,17 & 81,27 & 72,26 & $-0,3337$ & $-0,41$ & 0,0809 & 0,14 \\
\hline
\end{tabular}

$\mathrm{a}=\mathrm{y}^{1980} ; \mathrm{y}^{2007} ; \mathrm{b}$; \%v: variância anual; $\mathrm{R}^{2}$ : ; p: valor de p; DCV: doenças cardiovasculares; base: tendência das taxas calculadas sem correção dos registros de óbitos; ajust.: tendência das taxas ajustadas com redistribuição dos registros de óbitos mal definidos; ajust\&corrig.: tendência das taxas calculadas com os ajustes prévios mais correção de sub-registro de óbitos; DIC: doenças isquêmicas do coração; DCbV: doenças cerebrovasculares.

nos três níveis de correção, mostram bom ajuste ao modelo (Tabela 3).

Nas DIC, as diferenças são acentuadas, demonstrando que a metodologia de obtenção de taxas ajustadas $e$ corrigidas é capaz de elevar em quase três vezes a tendência de declínio nas taxas de mortalidade em Manaus para homens e em quase 4,5 vezes para mulheres, quando comparadas às taxas não corrigidas. Enquanto entre homens o percentual de variação anual se eleva de $-2,32 \%$ para $-2,73 \%$ (incremento de $17,7 \%)$, entre mulheres essa variação é de $-1,96 \%$ para $-2,76 \%$ (incremento de $40,8 \%)$, com tendência de queda significativa $(P<0,01)$. O valor de $\mathrm{R}^{2}$ mostra tendência de queda mais forte para as taxas ajustadas e corrigidas, para ambos os sexos (Tabela 3).
Se, nas taxas de mortalidade por DCV e DIC não há mudança no perfil da tendência, para as taxas de mortalidade por $\mathrm{DCbV}$ isso não é observado. Os valores $\mathrm{de} b$ diminuem continuamente entre as taxas base, as taxas corrigidas e as taxas corrigidas e ajustadas, para ambos os sexos (Figura 3). O percentual de variação anual $(\% v)$ também é menor, à medida que se observam as taxas com maior nível de correção (das taxas base para as taxas ajustadas e taxas ajustadas e corrigidas), chegando a valores próximos de zero, em ambos os sexos, para as últimas (Tabela 3$)$. Os valores de $\mathrm{R}^{2}$ tendem também a zero, indicando tendência muito fraca de declínio nas retas lineares para as taxas com mais ajuste, em ambos os gêneros. Os valores $\mathrm{p}$ mostram que não há significância estatística para a tendên- cia de declínio das taxas de mortalidade por DCbV em Manaus, para ambos os sexos, considerando as taxas de mortalidade ajustadas e corrigidas.

\section{DISCUSSÃO}

Considerando que o sub-registro de óbitos e a ocorrência de alto percentual de óbitos com causa mal definida, no SIM, são ainda realidade (29), buscou-se, neste artigo, ampliar a fidedignidade das taxas de mortalidade por doenças do coração.

A redistribuição de óbitos por causas mal definidas é um importante método de correção do SIM, pois atenua a influência que tais óbitos exercem sobre as taxas de mortalidade pelos demais grupos de causas definidas. 
A estimativa dos fatores de correção para o sub-registro de óbitos, por meio de técnicas demográficas indiretas, requer que as populações sob estudo apresentem determinadas características, como fecundidade constante e variação linear da mortalidade $(15,20,21)$, o que não é verdadeiro para o Brasil como um todo. Mesmo assim, devido ao sub-registro em muitas unidades federadas do Brasil, esses métodos têm sido utilizados para estimar o número de óbitos esperados no país (19) e também adotados como base das estimativas do IBGE, para avaliar a cobertura de óbitos. A comparação dos dados do SIM com as estimativas de óbitos do IBGE, representados pelo indicador "razão de óbitos informados e estimados", disponível no DATASUS (31), é o método em destaque para avaliar a qualidade dos dados de mortalidade no país, por região e Estado, sendo esse indicador baseado nos métodos indiretos utilizados neste estudo.

Laurenti et al. (32) observaram que as estatísticas vitais brasileiras têm melhorado desde sua implantação e destacaram haver estímulos para a utilização de dados secundários em pesquisas científicas no Brasil (33), embora ainda existam muitas críticas quanto à qualidade da informação, a qual tem se mostrado desigual entre regiões brasileiras (8) os piores indicadores estão nas regiões Norte e Nordeste.

Ao se utilizarem dados de um município do Norte do país, demonstrou-se ser possível corrigir as taxas, tornando-as mais acuradas, o que permite descrever a mortalidade cardiovascular no Município de Manaus. Estudos que utilizam técnicas de correção de sub-registro de óbitos não são comuns no Brasil. Destacam-se os artigos de Paes (14, 15), com mensuração do percentual de $\mathrm{C}$ dos dados de óbitos em cada região e Estado do país. Nos países desenvolvidos, as taxas de mortalidade por DCV são declinantes desde os anos 1960, mormente nos Estados Unidos, onde esse fenômeno foi inicialmente percebido (34). Razões atribuíveis a essa queda são conflitantes (35). No Brasil, essa tendência vem sendo descrita desde os anos 1980 (6), com 20 anos de defasagem em relação aos países desenvolvidos. No país, foi observado o declínio não só das taxas de mortalidade por DCV, como também de seus principais subgrupos, porém não há homogeneidade no Brasil (36).

Observa-se tendência de queda importante nas taxas ajustadas e corrigidas de mortalidade por DCV, em ambos os sexos em Manaus, de 1980 a 2007. Apesar da pequena diminuição dessa tendência nas taxas de mortalidade com maior grau de correção, os resultados confirmam os achados para os dados posteriores a 1980, já apontados para essa cidade por Mansur et al. (37) e Cesse et al. (38). É importante notar que, para as taxas ajustadas de mortalidade por DCV, observou-se aumento na tendência de declínio de óbitos para ambos os sexos. Isso demonstra mudança no comportamento das tendências de mortalidade cardiovascular em Manaus, quando corrigidos os dados secundários, de forma parcial ou mais abrangente, revendo códigos-lixo e subregistro de óbitos.

Esses achados demonstram que, apesar das DCV serem a principal causa de óbitos na cidade, observou-se tendência de declínio. Isso pode, em parte, ser reflexo do maior acesso da população a procedimentos que diminuem a letalidade, como também ao melhor controle dos fatores de risco (1).

As tendências para DIC também indicam queda das taxas, o que confirma achados de Mansur et al. (37) para 1980 a 1998. Neste estudo, a maior queda das taxas entre as mulheres não foi registrada por Mansur et al. (37), que creditaram a maior tendência de declínio aos homens. Porém, semelhante aos achados deste trabalho, maior tendência de declínio das taxas de mortalidade por DIC entre as mulheres já havia sido observada em estudos realizados somente na cidade de São Paulo (39) e no Brasil como um todo (40).

Os achados no presente estudo quanto às taxas de mortalidade por $\mathrm{DCbV}$ são preocupantes, por haver tendência de estabilidade das mesmas em ambos os sexos, enquanto há declínio das taxas para DIC. Esses achados divergem de recente estudo publicado (40), que demonstrou, para o Brasil como um todo, uma mudança na última década, na qual as DIC superavam as DCbV como principal causa de óbitos. Em Manaus, os dados ajustados e corrigidos mostram que as taxas de mortalidade por $\mathrm{DCbV}$ eram quase o dobro das taxas por DIC, entre os homens ao fim da série, em 2007. Entre as mulheres, as taxas de mortalidade por $\mathrm{DCbV}$, nesse mesmo ano, foram 2,4 vezes maiores do que as taxas por DIC.

Guimarães (41) assinalou que as maiores taxas de $\mathrm{DCbV}$ em regiões menos desenvolvidas originam-se do inadequado controle dos fatores de risco para DCV, particularmente a hipertensão arterial sistêmica. Em Manaus, as deficiências na implantação do programa governamental - sistema informatizado nacional de cadastro e acompanhamento de portadores de diabetes e hipertensão nas Unidades Básicas de Saúde (Sistema de Cadastramento e Acompanhamento de Hipertensas e Diabéticos-HiperDia), em conjunto com falhas na estruturação do Programa de Saúde da Família (PSF) (42), podem justificar esses achados.

Embora os resultados deste estudo sejam favoráveis à compreensão da realidade da mortalidade por DCV, cabem ressaltar as seguintes limitações:

- a falta de dados de migração impossibilita estimar a população de áreas pequenas com precisão. Entretanto, considera-se que a subestimação das taxas não compromete esses achados, pois pode exisitr um problema maior não relacionado à notificação do óbito, mas à sua qualidade;

- a impossibilidade de se conhecer o percentual de óbitos de cada capítulo da CID alocado como mal definido, obriga-nos à distribuição proporcional dos óbitos. Todavia, essa técnica contribui para a subestimação de taxas de mortalidade por DCV, visto que não há como afirmar que distribuição dos óbitos seria igual entre os capítulos da CID;

- o uso de técnicas indiretas, para correção de sub-registro de óbitos, idealmente, deveria pressupor populações fechadas e com taxas de crescimento estáveis.

Constatou-se, neste estudo, que as taxas ajustadas e corrigidas para DCV não mostraram grande impacto em relação às taxas base em ambos os sexos, ainda que apresentem pequena diminuição na tendência de declínio. Para as DIC e $\mathrm{DCbV}$, as taxas ajustadas e corrigidas mostraram grande impacto em relação às taxas base e também em relação às taxas ajustadas para as causas mal definidas.

A correção de sub-registros de óbitos por DCV, DIC e DCbV em Manaus proporcionou conhecimento das taxas de mortalidade padronizadas, permitindo avaliar adequadamente as tendências. Portanto, com essa correção, foi possível identificar excesso de óbitos que não havia sido computado, gerando taxas de mortalidade ajustadas e corrigidas mais confiáveis. 


\section{REFERÊNCIAS}

1. Malta DC, Cezário AC, Moura L, Morais Neto OL, Silva Junior JB. A construção da vigilância e prevenção das doenças crônicas não transmissíveis no contexto do Sistema Único de Saúde. Epidemiol Serv Saude. 2006;15(3): 47-65.

2. World Health Organization (WHO) [Internet]. Cardiovascular diseases (CVDs). Fact sheet $\mathrm{N}^{\circ}$ 317. 2009. Disponível em: http://www. who.int/mediacentre/factsheets/fs317/en/ print.html Acessado em 5 de setembro de 2012.

3. Weisz G, Olszynko-Gryn J. The theory of epidemiologic transition: the origins of a citation classic. J Hist Med Allied Sci. 2010;65(3): 287-326.

4. Levi F, Chatenoud L, Bertuccio P, Lucchini F, Negri E, La Vecchia C. Mortality from cardiovascular and cerebrovascular diseases in Europe and other areas of the world: an update. Eur J Cardiovasc Prev Rehabil. 2009;16(3):333-50.

5. Mansur AdeP, de Souza Mde F, Timerman A, Avakian SD, Aldrighi JM, Ramires JA. Trends in the risk of death from cardiovascular, cerebrovascular and ischemic diseases in thirteen States of Brazil from 1980 to 1998. Arq Bras Cardiol. 2006;87(5):641-8.

6. Soares GP, Brum JD, Oliveira GM, Klein CH, Silva NAS. Mortalidade por todas as causas e por doenças cardiovasculares em três estados do Brasil, 1980 a 2006. Rev Panam Salud Publica. 2010;28(4):258-66.

7. Brasil. Ministério da Saúde. Departamento de Informática do Sistema Único de Saúde (DATASUS) [Internet]. Motalidade - Brasil. Disponível em: http://tabnet.datasus. gov.br/cgi/tabcgi.exe?sim/cnv/obt10uf.def Acessado em 5 de setembro de 2012.

8. Jorge MH, Laurenti R, Gotlieb SL. Análise da qualidade das estatísticas vitais brasileiras: a experiência de implantação do SIM e do SINASC. Cien Saude Colet. 2007;12(3):643-54.

9. Centro Colaborador da OMS para a Classificação de Doenças em Português (CBCD) [Internet]. Classificação Estatística Internacional de Doenças e Problemas Relacionados à Saúde - CID-10. Disponível em: http:// www.datasus.gov.br/cid10/v2008/cid10. $\mathrm{htm}$ Acessado em 5 de setembro de 2012

10. Curioni $C$, Cunha $C B$, Veras RP, André $C$. The decline in mortality from circulatory diseases in Brazil. Rev Panam Salud Publica. 2009;25(1): 9-15.

11. Marinho de Souza MF, Alencar AP, Malta DC, Moura L, Mansur AP. Análise de séries temporais da mortalidade por doenças isquêmicas do coração e cerebrovasculares, nas cinco regiões do Brasil, no período de 1981 a 2001. Arq Bras Cardiol. 2006;87(6):735-40.

12. Oliveira GM, Silva NA, Klein CH. [Balanced cardiovascular disease mortality from 1980 to 1999-Brazil]. Arq Bras Cardiol. 2005;85(5): 305-13. Article in Portuguese.

13. Cavalini LT, Ponce de Leon AC. Correção de sub-registros de óbitos e proporção de internações por causas mal definidas. Rev Saude Publica. 2007;41(1):85-93.

14. Paes NA. Avaliação da cobertura dos registros de óbitos dos estados brasileiros em 2000. Rev Saude Publica. 2005;39(6):882-90.

15. Paes NA. [Quality of death statistics by unknown causes in Brazilian states]. Rev Saude Publica. 2007;41(3):436-45. Article in Portuguese.
16. Schramm JMA, Oliveira AF, Leite IC, Valente JG, Gadelha AMJ, Portela MC, et al. Transição epidemiológica e o estudo de carga de doença no Brasil. Cien Saude Colet. 2004;9:897-908.

17. Instituto Brasileiro de Geografia e Estatística (IBGE). Banco de Dados Agregados. Sistema IBGE de Recuperação Automática (SIDRA) [Internet]. Índice Nacional de Preços do Consumidor Amplo — agosto de 2012. Disponível em: http://www.sidra.ibge.gov.br/bda/default.asp Acessado em 5 de setembro de 2012.

18. Brasil. Ministério da Saúde. Departamento de Informática do Sistema Único de Saúde (DATASUS) [homepage da Internet]. Disponível em: http://www2.datasus.gov.br/ DATASUS/index.php?area=0206 e http:// tabnet.datasus.gov.br/tabdata/sim/dados/ indice.htm Acessado em 15 de setembro de 2009.

19. Gadelha A, Leite IC, Valente JG, Schramm JM, Portela MC, Campos MR [Internet]. Relatório Final do Projeto Estimativa da Carga de Doença do Brasil - 1998. Rio de Janeiro: ENSP/Fiocruz-FENSPTEC, 2002. Disponível em: http:// www4.ensp.fiocruz.br/projetos/carga/down loads1.htm Acessado em 5 de setembro de 2012.

20. Brass W. Methods for estimating fertility and mortality from limited and defective data. In: Hill C, editor. North Caroline: Caroline Population Center. Laboratories for Population Studies; 1975.

21. Preston S, Coale AJ, Trussell J, Weinstein $M$. Estimating the completeness of reporting of adult deaths in populations that are approximately stable. Popul Index. 1980;46(2): 179-202.

22. United Nations (ONU). Department of Economic and Social Affairs. Population Division [Internet]. MANUAL X: Indirect Techniques for Demographic Estimation. United Nations publication, Sales No. E.83.XIII.2; 1983. Disponível em: http://www.un.org/esa/popu lation/publications/Manual_X/Manual_X. $\mathrm{htm}$ Acessado em 5 de setembro de 2012.

23. Silvi J. On the estimation of mortality rates for countries of the Americas. Epidemiol Bull. 2003;24(4):1-5.

24. Laurenti R, Mello Jorge MHP, Lebrão ML, Gotlieb SLD. Estatísticas de saúde. 2 ed. São Paulo: EPU; 2005.

25. Instituto Brasileiro de Geografia e Estatística (IBGE). Censo Demográfico 1991. Disponível em: http://www.ibge.gov.br/home/esta tistica/populacao/censodem/default.shtm Acessado em 15 de setembro de 2009

26. Murray CJL, Lopez AD. The Global Burden of Disease: a comprehensive assessment of mortality and disability from diseases, injuries, and risk factors in 1990 and projected to 2020. Cambridge, MA: Harvard University Press on behalf of the World Health Organization and the World Bank; 1996.

27. Mathers CD, Vos T, Stevenson C [Internet]. The burden of disease and injury in Australia-Summary Report. Canberra: Australian Institute of Health and Welfare; 1999. Disponível em: www.aihw.gov. $\mathrm{au} /$ WorkArea/DownloadAsset.aspx?id= 6442459196 Acessado em 5 de setembro de 2012.

28. NZHIS [Internet]. A complete list of oneto-one backwards mappings ICD-10 to ICD9. Ministry of Health/New Zealand, 2000.
Disponível em: http://www.nzhis.govt.nz/ moh.nsf/pagesns/254 Acessado em 2 de setembro de 2009.

29. Laurenti R, Mello Jorge MHP. O Sistema de Informações sobre Mortalidade: passado, presente e futuro. São Paulo: Centro Colaborador da OMS para a Família de Classificações em Português; 2006.

30. Paes NA, Albuquerque MEE. Avaliação da qualidade dos dados populacionais e cobertura dos registros de óbitos para as regiões brasileiras. Rev Saude Publica. 1999:33(1):33-43.

31. Brasil. Ministério da Saúde, Departamento de Informática do Sistema Único de Saúde (DATASUS). Rede Interagencial de Informações para a Saúde. A.18 Razão entre óbitos informados e estimados [homepage da Internet]. Disponível em: http://tabnet.datasus. gov.br/cgi/idb2009/a1801b.htm Acessado em 15 de setembro de 2009.

32. Laurenti R, Jorge MHPM, Lebrão ML, Gotlieb SLD, Almeida MF. Editorial Especial. Estatísticas vitais: contando os nascimentos e as mortes. Rev Bras Epidemiol. 2005;8(2):108-10.

33. Drumond EF, Machado CJ, Vasconcelos MR, França E. Utilização de dados secundários do SIM, Sinasc e SIH na produção científica brasileira de 1990 a 2006. Rev Bras Estud Popul. 2009;26(1):7-19.

34. Centers for Disease Control and Prevention (CDC). Prevalence of coronary heart disease-United States, 2006-2010. MMWR Morb Mortal Wkly Rep. 2011;60(40):1377-81.

35. Mathers CD. Health expectancies: an overview and critical appraisal. In: Murray CJL, Salomon JA, Mathers CD, Lopez AD, editors. Summary measures of population health. Genebra: WHO; 2002. Pp. 177-204.

36. Lotufo PA. Por que não vivemos uma epidemia de doenças crônicas: o exemplo das doenças cardiovasculares? Cien Saude Colet. 2004;9(4):844-7.

37. Mansur AP, Souza MF, Timmermann A, Ramires JAF. Trends of the risk of death due to circulatory, cerebrovascular, and ischemic heart diseases in 11 Brazilian capitals from 1980 to 1998. Arq Bras Cardiol. 2002;79(3): 269-84.

38. Cesse EAP, Carvalho EF, Souza WV, Luna CF. Tendência da mortalidade por doenças do aparelho circulatório no Brasil: 1950 a 2000. Arq Bras Cardiol. 2009;93(5):490-7.

39. Farias N, Souza JM, Laurenti R, Alencar SM. Mortalidade cardiovascular por sexo e faixa etária em São Paulo, Brasil: 1996 a 1998 e 2003 a 2005. Arq Bras Cardiol. 2009;93(5):498-505.

40. Mansur AP, Lopes AIA, Favarato D, Avakian SD, César LAM, Ramires JAF. Transição epidemiológica da mortalidade por doenças circulatórias no Brasil. Arq Bras Cardiol. 2009;93(5):468-510.

41. Guimarães AC. Hypertension in Brazil. J Hum Hypertens. 2002;16 Suppl 1;57-10.

42. Silva NC, Garnelo L, Giovanella L. Extensão de cobertura ou reorganização da Atenção Básica? A trajetória do Programa de Saúde da Família de Manaus-AM. Saude Soc. 2010;19(3):592-604.

Manuscrito recebido em 8 de dezembro de 2011. Aceito em versão revisada em 30 de julho de 2012 
ABSTRACT Objective. Identify the impact of corrective techniques in the mortality rate trends for cardiovascular disease, ischemic heart diseases, and cerebrovascular diseases, in the City of Manaus, State of Amazonas, Brazil, between 1980 and 2007.

\section{Cardiovascular mortality and impact of corrective techniques for dealing with underreported and ill-defined deaths}

Methods. Data were obtained from the Unified Health System's Information Technology Department and the following steps undertaken: (1) proportional redistribution of death records, but without taking into account age and/or sex; (2) redistribution of ill-defined deaths among those whose cause is known; (3) treatment and reallocation of so-called "junk codes" in cardiology; and (4) correction of underrecordings of deaths by indirect techniques. Records treated in steps 1 and 2, and steps 3 and 4 together, generated base rates, adjusted rates, and adjusted and corrected rates, respectively, which were analyzed according to sex and age cohort; subsequently, they were standardized by the direct method. Simple linear regression was used for trend analysis.

Results. The Mortality Information System's raw data underestimated death from heart diseases, mainly starting in the year 2000. With regard to the trend over time, the adjusted and corrected rates, compared to the base rates, pointed to the following: attenuation of the downward trend for cardiovascular diseases as a whole; accentuation of the downward trend for ischemic heart diseases; and stability in the rates for cerebrovascular diseases during the period.

Conclusions. This correction made it possible to identify an excess of deaths that had not been computed, generating more reliable adjusted and corrected death rates.

Key words Cardiovascular diseases; vital statistics; mortality; underregistration; mortality registries; information systems; Brazil. 